\title{
Avaliação temporal das características funcionais de espécies arbóreas em fitofisionomias da transição Cerrado-Amazônia, Mato Grosso, Brasil
}

\author{
Giovana Zilli ${ }^{1}$ \\ Beatriz Schwantes Marimon ${ }^{1}$ \\ Marco Antonio Camillo de Carvalho ${ }^{1,2}$ \\ Fernando Landa Sobral ${ }^{3}$ \\ Ben Hur Marimon-Junior ${ }^{1}$ \\ ${ }^{1}$ Programa de Pós-graduação em Ecologia e Conservação, \\ Universidade do Estado de Mato Grosso, Campus de Nova Xavantina \\ Caixa Postal 08, CEP 78.690-000, Nova Xavantina - MT, Brasil \\ ${ }^{2}$ Universidade do Estado de Mato Grosso, Campus de Alta Floresta \\ Alta Floresta - MT, Brasil \\ ${ }^{3}$ Programa de Pós-graduação em Ecologia e Evolução \\ Universidade Federal de Goiás, Goiânia - GO, Brasil \\ * Autor para correspondência: \\ biamarimon@unemat.br
}

Submetido em 24/11/2013

Aceito para publicação em 30/07/2014

\section{Resumo}

O estudo objetivou investigar possíveis variações temporais das características funcionais de espécies arbóreas de três fisionomias de Cerrado. Foram analisadas 195 espécies, sendo 88 em cerrado típico, 89 em cerradão e 105 em mata de galeria, com 87 espécies comuns em pelo menos duas fisionomias. O estudo se baseou em listas de levantamentos florísticos e fitossociológicos ao longo de 12 anos. Foi registrada variação na diversidade funcional para o cerrado típico e o cerradão ao longo do tempo, enquanto a mata de galeria permaneceu constante. As três comunidades apresentaram variações na riqueza de espécies e número de indivíduos ao longo dos inventários, provavelmente atribuídas a eventos de seca e fogo que ocorreram no período de estudo. Estes resultados revelam grande estabilidade funcional da mata de galeria frente às variações ambientais, florísticas e fitossociológicas ocorridas no período. As causas das mudanças verificadas no cerrado típico e no cerradão são ainda incertas, mas podem estar relacionadas à maior instabilidade hídrica destas comunidades em relação à mata de galeria, onde as árvores têm melhor acesso à água no solo ao longo do ano.

Palavras-chave: Comunidade; Diversidade funcional; Fogo; Seca

\section{Abstract}

Evaluation of temporal variation in functional characteristics of arboreal species in CerradoAmazonia transition zone physiognomies, Mato Grosso state, Brazil. This study investigated the possibility of temporal variation in functional characteristics of arboreal species in three Cerrado vegetation physiognomies. 
We analyzed 195 species in total, including 88 in typical cerrado, 89 in cerradão and 105 in gallery forest, with 87 species common in at least two physiognomies. The study was based on lists provided by floristic and phytosociological surveys conducted over 12 years. We observed temporal variation in functional diversity values for typical cerrado and cerradão, while the gallery forest remained constant. The arboreal communities in all three physiognomies showed temporal variation in species richness and abundance, which is likely attributed to drought and fire events that occurred during the study. The lack of temporal change in functional diversity, species richness, and abundance in the gallery forest suggests higher functional stability during periods of environmental change. The causes of the functional changes observed in the typical cerrado and cerradão remain uncertain, but may be related to greater seasonality of soil water availability compared to the gallery forest, where trees have increased access to soil water throughout the year.

Key words: Community; Drought; Fire; Functional Diversity

\section{Introdução}

Estudos de diversidade funcional em comunidades vegetais permitem elucidar processos ecológicos de estruturação e funcionamento dessas comunidades frente às mudanças ambientais. A diversidade funcional traz informações importantes sobre as características das espécies nas comunidades (PETCHEY; GASTON, 2006; MOUCHET et al., 2010) e, portanto, explica melhor a estrutura e o funcionamento dos ecossistemas (PETCHEY; GASTON, 2006; MOUCHET et al., 2010; CIANCIARUSO et al., 2012; 2013; DANTAS et al., 2013). Os padrões de diversidade dos traços funcionais podem ser usados como fortes argumentos para a conservação dos habitats (MAYFIELD et al., 2006), que tem sido fomentados por estudos de diversidade funcional em comunidades vegetais do Cerrado (e.g. CIANCIARUSO et al., 2009; 2010, 2013; BATALHA et al., 2011; DANTAS et al., 2013). Estes argumentos são suficientemente sólidos para promover a conservação de processos e serviços do ecossistema que dependem da integridade das comunidades e do seu conjunto funcional de espécies (CIANCIARUSO et al., 2012; 2013).

Os estudos de diversidade funcional tornam-se especialmente importantes atualmente, tendo em vista que o Cerrado é um hotspot para a conservação, devido à alta pressão antrópica a que vem sendo submetido nas últimas décadas (KLINK; MACHADO, 2005). As queimadas estão entre os maiores distúrbios que as formações vegetais de Cerrado enfrentam devido ao clima estacional, com períodos de seca de até seis meses (OLIVEIRA, 2008; CIANCIARUSO et al., 2010). Esta estacionalidade climática favorece a ocorrência de queimadas que podem ser naturais ou antrópicas
(COUTINHO, 1990) conduzindo, ao longo do tempo, às adaptações vegetativas e reprodutivas das plantas para persistirem nesse ambiente (OLIVEIRA, 2008; CIANCIARUSO et al., 2010). Neste caso, tanto a presença quanto a exclusão do fogo, além da frequência ou época das queimadas podem alterar a estrutura e a composição florística de uma comunidade vegetal (MOREIRA, 2000). Apesar das pressões que ocorrem no Cerrado, ele é resiliente ao fogo e a outros distúrbios (HOFFMANN, 2000; ELIAS et al., 2013), pois suas espécies apresentam alta capacidade de regeneração natural através do banco de sementes ou por rebrotas (HOFFMANN, 2005).

Considerando a escassez de estudos que avaliaram a variação temporal de características funcionais, este trabalho teve como objetivo investigar possíveis variações temporais da diversidade funcional em três comunidades vegetais da transição Cerrado-Amazônia, na região Leste de Mato Grosso. Neste contexto, elaboramos a seguinte pergunta: o valor de diversidade funcional das comunidades varia entre os períodos analisados? Esperamos que sim, pois mudanças ambientais, como fogo e seca, podem influenciar a diversidade funcional (CIANCIARUSO et al., 2010; 2012; 2013).

\section{Material e Métodos}

\section{Área de estudo}

O estudo foi desenvolvido em 50 parcelas permanentes de $10 \times 10 \mathrm{~m}$, estruturadas de maneira aleatória, totalizando 0,5 ha em cada área, em três 
fisionomias: mata de galeria, cerradão e cerrado típico, no Parque Municipal do Bacaba, na Universidade do Estado de Mato Grosso, Campus de Nova Xavantina, região leste de Mato Grosso, na transição entre o Cerrado e a Amazônia (MARIMON et al., 2014). O parque apresenta uma área de aproximadamente 500 ha e engloba diversas fisionomias, desde formações florestais, com fragmentos de cerradão e mata de galeria (MARIMON et al., 2001; MARIMON-JUNIOR; HARIDASAN, 2005), até formações savânicas, como cerrado rupestre e cerrado típico (ABAD; MARIMON, 2008; MEWS et al., 2011).

O parque apresenta altitude que varia entre $250 \mathrm{a}$ $300 \mathrm{~m}$ e clima do tipo tropical de savana (Aw segundo Köppen), com temperatura média anual de $25^{\circ} \mathrm{C}$ e precipitação média anual de $1.550 \mathrm{~mm}$, com duas estações definidas, uma seca de abril a setembro e outra chuvosa de outubro a março (ABAD; MARIMON, 2008). Dois eventos de seca (anomalias) relacionados com elevadas taxas de mortalidade de árvores foram registrados para a região, um em 2005 e outro em 2010 (MARENGO et al., 2008; LEWIS et al., 2011). Em julho de 2001 foi registrada uma queimada no parque que atingiu cerca de $70 \%$ das parcelas da mata de galeria, $20 \%$ do cerrado típico e nenhuma do cerradão. Em setembro de 2008 foi registrada outra queimada que atingiu as três áreas de estudo em proporções similares, cerca de $30 \%$ das parcelas em cada área.

\section{Coleta de dados}

As espécies avaliadas no presente estudo foram amostradas em levantamentos fitossociológicos e florísticos realizados anteriormente, cujas listas e características estruturais foram publicadas em Franczak et al. (2011), Marimon-Junior e Haridasan (2005), Marimon et al. (2001; 2002; 2010), Mews et al. (2011) e Miguel et al. (2011a; 2011b). Os dados de temperatura média mensal e precipitação foram coletados na Estação Meteorológica de Nova Xavantina-MT, Instituto Nacional de Meteorologia (OMM: 83319).

As coletas em campo foram realizadas em visitas quinzenais de fevereiro de 2011 a março de 2012. Para a determinação das características funcionais, foram coletados frutos e sementes das espécies listadas nos levantamentos citados, e realizadas observações em campo das demais características funcionais, como dispersão e polinização. As espécies que não foram encontradas em estágio reprodutivo tiveram as características funcionais e demais informações adquiridas a partir de consultas em material bibliográfico (e.g. LORENZI, 1992, 1998; BARROSO et al., 1999; CARVALHO, 2003) e sites da internet (e.g. http:// www.nybg.org; http://science.mnhn.fr/institution/ mnhn/search; http://www.frutosatrativosdocerrado. bio. br). Foram coletados 15 frutos e 15 sementes de cada espécie e medidos com paquímetro ao longo de seu maior eixo. $\mathrm{O}$ material foi seco em estufa a $70^{\circ} \mathrm{C}$ até obtenção de peso constante e em seguida pesado em balança de precisão para determinar a massa seca. Os traços funcionais analisados e seus respectivos significados ecológicos foram baseados em Cornelissen et al. (2003) e Cianciaruso et al. (2009) (Tabela 1).

TABELA 1: Traços funcionais utilizados para calcular o valor de diversidade funcional do cerrado típico, cerradão e mata de galeria. Sendo: Cat. $=$ categórico. Baseado em Cornelissen et al. (2003) e Cianciaruso et al. (2009).

\begin{tabular}{lcl}
\hline \multicolumn{1}{c}{ Traço } & Unidade & \multicolumn{1}{c}{ Significado ecológico } \\
\hline Tamanho do fruto & $\mathrm{mm}$ & $\begin{array}{l}\text { Vigor competitivo, capacidade de } \\
\text { re/colonização } \\
\text { Vigor competitivo, capacidade de } \\
\text { Tamanho da semente }\end{array}$ \\
mm & $\mathrm{g}$ & $\begin{array}{l}\text { Vigor colonização } \\
\text { re/colonização }\end{array}$ \\
Peso do fruto & $\mathrm{g}$ & $\begin{array}{l}\text { Vigor competitivo, capacidade de } \\
\text { re/colonização }\end{array}$ \\
Peso da semente & $\mathrm{Cat}$. & $\begin{array}{l}\text { Capacidade de re/colonização } \\
\text { Tipo de dispersão }\end{array}$ \\
Tipo de polinização & Cat. & $\begin{array}{l}\text { Capacidade de re/colonização } \\
\text { Vigor competitivo, capacidade de }\end{array}$ \\
Tipo de fruto & Cat. & $\begin{array}{l}\text { re/colonização } \\
\text { Vigor competitivo, resposta a }\end{array}$ \\
Forma de vida & Cat. & \begin{tabular}{l} 
distúrbios \\
\hline
\end{tabular}
\end{tabular}

Foram amostrados todos os indivíduos com diâmetro $\geq 5 \mathrm{~cm}$ na altura de $30 \mathrm{~cm}$ do solo (DAS ${ }_{30 \mathrm{~cm}}$ ) para o cerrado típico e o cerradão e DAP (diâmetro à altura do peito) $\geq 5 \mathrm{~cm}$ na mata de galeria em todos os levantamentos. Os inventários no cerrado típico foram realizados em 2002, 2006, 2008 e 2011; no cerradão em 
2002, 2005, 2008 e 2010 e na mata de galeria em 1999, 2006 e 2011. As listas publicadas das áreas (MARIMON et al., 2002; MARIMON-JUNIOR; HARIDASAN, 2005; FRANCZAK et al., 2011; MEWS et al., 2011; MIGUEL et al., 2011a; 2011b) apontaram 129 espécies na mata de galeria em 1999, 135 espécies em 2006 e 132 em 2011. No cerradão foram 77 espécies em 2002, 95 em 2005, 84 em 2008 e 82 em 2010. No cerrado típico foram amostradas 77 espécies em 2002, 80 em 2006, 82 em 2008 e 88 em 2011.

\section{Análise de dados}

Para avaliar a similaridade florística entre os anos em cada área foram utilizados o índice de Sørensen (CCs), baseado na presença e ausência das espécies, e o índice de Morisita (IM), que é quantitativo e baseia-se na abundância das espécies (BROWER; ZAR, 1977). Os valores destes índices variam entre 0 e 1 , sendo que valores acima de 0,5 indicam elevada similaridade florística (KENT; COKER, 1992).

A quantificação da diversidade funcional se baseou nos fundamentos de uma análise de agrupamento e, por isso, consistiu em: i) elaborar uma matriz de características, ii) converter a matriz de características em uma matriz de distância, iii) realizar o agrupamento da matriz de distância para produzir um dendrograma e, iv) calcular a distância média entre todos os pares de espécies que ocorreram em determinada assembleia. Portanto, inicialmente foi construída uma matriz de características, com as espécies dispostas em linhas e as características coletadas em campo (Tabela 1) dispostas em colunas. A partir da matriz de características, foi construída uma matriz de distância utilizando uma generalização da distância de Gower (PAVOINE et al., 2009), dedicada ao tratamento de dados mistos (e.g. quantitativos e qualitativos). Em seguida, foi produzido o dendrograma funcional através do método de agrupamento pela média (UPGMA), com o qual foi calculada a diversidade funcional das comunidades (cerrado típico, cerradão e mata de galeria).

Para quantificar a diversidade funcional observada em cada uma das comunidades foi utilizada a Distância Média de Pares (DMP) (WEBB, 2000), uma medida de diversidade biológica independente da riqueza de espécies e amplamente utilizada para investigar os mecanismos evolutivos e ecológicos responsáveis pela montagem das assembleias (e.g. KEMBEL, 2009; MCEWEN; VAMOSI, 2010). Originalmente a medida DMP foi proposta para quantificar a distância filogenética entre espécies (WEBB, 2000). Entretanto, qualquer índice baseado em distâncias entre espécies pode ser aplicado tanto para o cálculo da diversidade filogenética quanto para o cálculo da diversidade funcional (PAVOINE; BONSALL, 2011). Portanto, no presente trabalho, a DMP quantifica a distância funcional de pares entre cada uma das espécies na comunidade e, então, indica a distância funcional média entre todos os táxons (WEBB, 2000). Para testar se a diversidade funcional, a riqueza de espécies e o número de indivíduos diferiram significativamente ao longo dos anos em cada fisionomia, foi realizada uma análise de variância de medidas repetidas, através do software STATISTICA (STATSOFT, 2007).

Para testar se a diversidade funcional em cada comunidade foi diferente da esperada ao acaso, foram utilizados modelos nulos para comparar os resultados observados de DMP com o esperado para 1000 comunidades construídas aleatoriamente, com base no banco total de espécies presente em cada comunidade. Ou seja, para calcular os modelos nulos foram utilizados três bancos de espécies, cada um referente a uma distinta fisionomia. Para construir as comunidades simuladas utilizamos um modelo nulo aleatorizando as espécies presentes no dendrograma funcional, calculando para cada repetição valores simulados de DMP. Isso significa que todas as comunidades simuladas mantém o mesmo número de espécies observado, embora possam ser compostas por qualquer espécie presente na fisionomia.

Em seguida, foi calculado o tamanho do efeito padronizado de DMP para cada comunidade em relação às respectivas distribuições aleatorizadas, usando a seguinte fórmula: $(Z)=\left(\mathrm{obs}_{\mathrm{DMP}}-\mathrm{rnd}_{\mathrm{DMP}}\right) / \mathrm{sd} \mathrm{rnd}_{\mathrm{DMP}}$, onde obs ${ }_{\text {DMP }}$ é o valor observado de DMP; $\operatorname{rnd}_{\mathrm{DMP}}$ é a média dos valores aleatorizados de DMP; e sd.rnd ${ }_{D M P}$ é o desvio padrão dos valores aleatorizados de DMP. Para testar se, na média, o tamanho dos efeitos padronizados encontrados para as comunidades de cerradão, mata 
de galeria e cerrado típico apresentaram desvios significativos a partir do esperado ao acaso, foi realizado um teste $t$ para uma única amostra, comparando a média dos valores de efeito padronizado em relação a uma média conhecida (média =0; veja CIANCIARUSO et al., 2012, para abordagem similar). Valores do tamanho do efeito padronizado maiores do que zero indicam diversidade funcional maior do que a esperada pelo acaso e valores menores do que zero indicam diversidade funcional menor do que a esperada pelo acaso (WEBB, 2000). As análises de diversidade funcional foram realizadas utilizando os pacotes "vegan", "ade4" e "picante" no ambiente computacional R (R DEVELOPMENT CORE TEAM, 2012).

\section{Resultados}

As três comunidades avaliadas apresentaram elevada similaridade na composição florística entre os anos, para ambos os índices de similaridade $(>0,89 \mathrm{em}$ todas as comparações). No total, foram amostradas e analisadas as características funcionais de 195 espécies, sendo 88 no cerrado típico, 89 no cerradão e 105 na mata de galeria, onde 87 foram comuns em pelo menos duas fisionomias e apenas 15 foram comuns entre as três fisionomias: Agonandra brasiliensis Miers ex Benth. \& Hook.f., Astronium fraxinifolium Schott, Bowdichia virgilioides Kunth, Curatella americana L., Dipteryx alata Vogel, Emmotum nitens (Benth.) Miers, Erythroxylum daphnites Mart., Guapira graciliflora (Mart. ex Schmidt) Lundell, Magonia pubescens A.St.Hil., Matayba guianensis Aubl., Mimosa laticifera Rizzini \& A.Mattos, Plathymenia reticulata Benth., Qualea multiflora Mart., Tachigali vulgaris L.G.Silva \& H.C.Lima e Xylopia aromatica (Lam.) Mart.

Durante o período analisado, o cerrado típico apresentou um valor médio de DMP, igual a 0,571 $( \pm 0,016)$. O valor de DMP aumentou ao longo dos anos (Figura 1A) como esperado, sendo a diferença significativa entre os anos de 2002 e 2011 (Figura 1A).

FIGURA 1: Média observada e desvio padrão de diversidade funcional, riqueza de espécies e número de indivíduos, nas comunidades lenhosas de cerrado típico, cerradão e mata de galeria, ao longo dos anos, Nova Xavantina-MT.
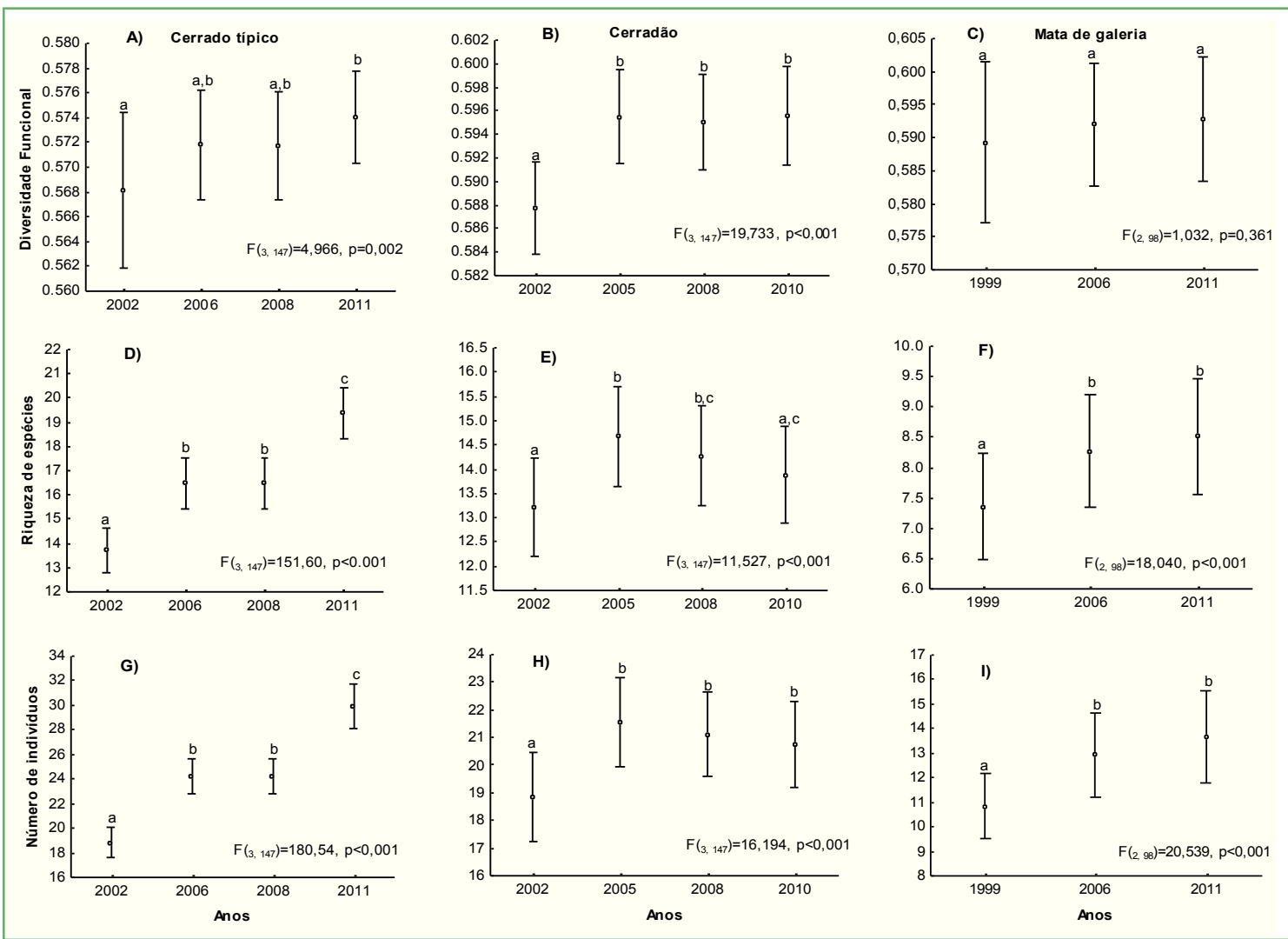
O cerradão apresentou ao longo dos anos um valor médio de DMP igual a 0,593 $( \pm 0,014)$ e, nesta fisionomia, o DMP também aumentou entre o primeiro e os demais anos (Figura 1B). A mata de galeria apresentou o valor médio de DMP igual a $0,591( \pm 0,036)$, sem apresentar mudanças ao longo do período avaliado (Figura 1C).

No geral, tanto os valores da riqueza de espécies (Figuras 1D e 1E), quanto do número de indivíduos (Figuras $1 \mathrm{G} \mathrm{e} \mathrm{H}$ ) do cerrado típico e do cerradão aumentaram ao longo dos anos, coincidindo com o aumento na diversidade funcional ( $p$ de Tukey $<0,001$ ). Entretanto, no cerradão, entre 2005 e 2010 a riqueza diminuiu, retornando aos valores registrados em 2002. Na mata de galeria, o aumento do número de indivíduos não coincidiu com o aumento na diversidade funcional, embora a riqueza (Figura 1F) e o número de indivíduos (Figura 1I) aumentaram significativamente ao longo dos anos. Entretanto, esta diferença foi observada apenas entre 1999 e os demais anos ( $p$ de Tukey $<0,001$; Figuras
1F e 1I). Também foi observado um aumento nas taxas de precipitação nas áreas de estudo em 2003, 2004 e 2006, bem como uma redução no ano de 2005. Além disso, houve aumento na média da temperatura em 2005, 2008 e 2009 (Figura 2).

A diversidade funcional das comunidades de cerradão e de mata de galeria não diferiu do acaso nos diferentes anos, pois todos os valores de Z, na média, não foram significativamente diferentes de zero (Figura 3). Isso indica que não podemos inferir sobre a atuação de filtros ambientais ou de processos, como similaridade limitante, na estruturação das comunidades estudadas. Por outro lado, no ano de 2011, a comunidade de cerrado típico apresentou diversidade funcional menor do que o esperado pelo acaso (valor de $\mathrm{Z}$ negativo), embora os anos anteriores também tenham apresentado valores de $\mathrm{Z}$ que estatisticamente não diferiram de zero (Figura 3).

FIGURA 2: Climatograma representativo para Nova Xavantina-MT entre os meses de janeiro de 1999 a dezembro de 2011, sendo: precipitação anual, $\bullet$ temperatura média do ar. Fonte: INMET, Estação Meteorológica de Nova Xavantina-MT (OMM: 83319).

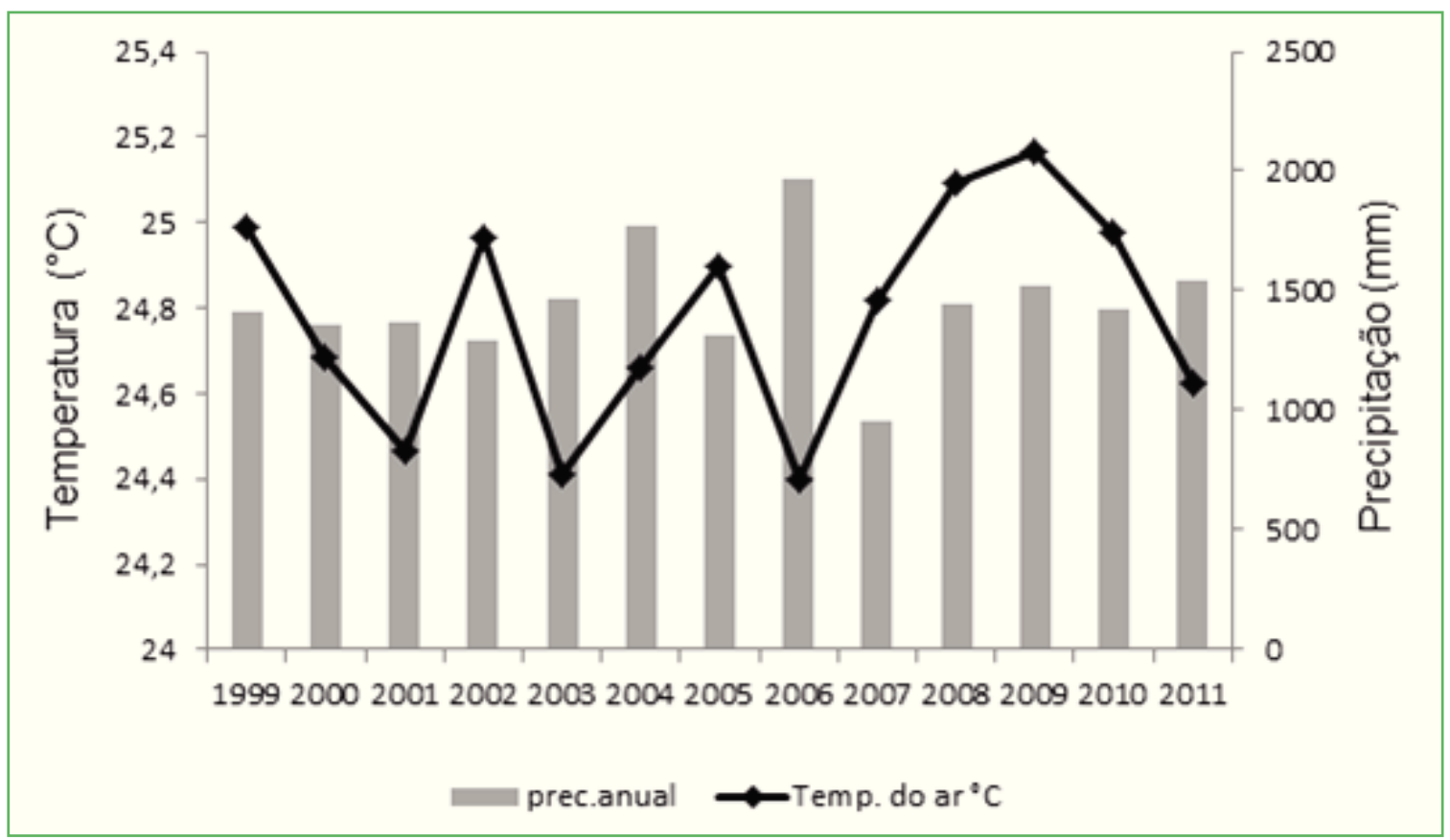


FIGURA 3: Tamanhos de efeito padronizados de diversidade funcional e seus intervalos de confiança de $95 \%$ para as comunidades de cerradão, mata de galeria e cerrado típico, Nova Xavantina-MT. O asterisco indica valor significativamente diferente de zero.

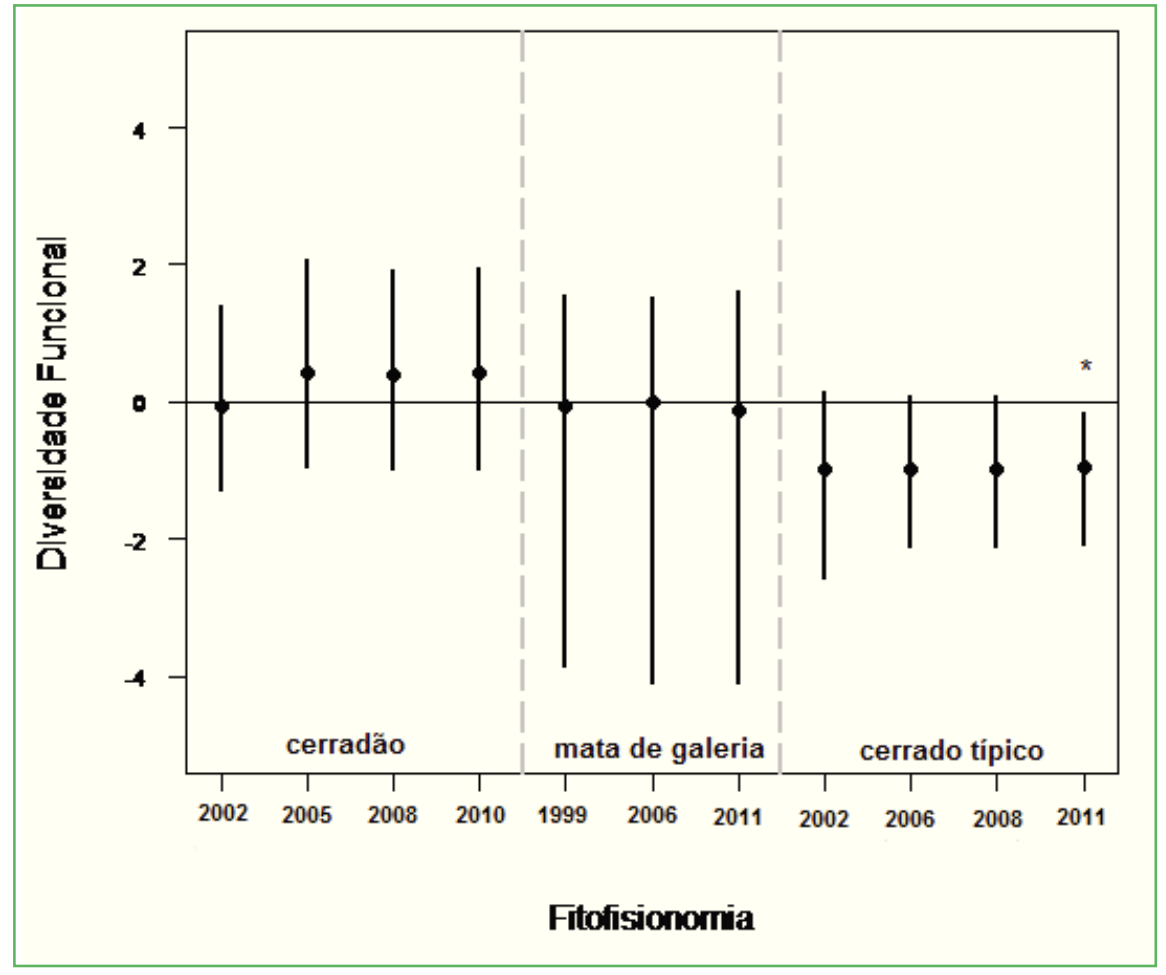

\section{Discussão}

Apesar da alta similaridade florística entre os anos, observada em cada comunidade, que pode indicar alta resiliência às perturbações de seca e queimada registradas no período, tanto a riqueza de espécies quanto o número de indivíduos das três comunidades sofreram alterações ao longo dos inventários. Neste caso, o aumento na riqueza de espécies pode aumentar a diversidade funcional (CIANCIARUSO et al., 2009) quando a espécie adicionada na comunidade não apresentar redundância funcional, isto é, a espécie adicionada apresenta características funcionalmente diferentes das existentes na comunidade (PETCHEY; GASTON, 2006).

O aumento na diversidade funcional do cerrado típico sugere que a passagem do fogo tenha atuado nesta área como um elemento de retroalimentação positiva, favorecendo o desenvolvimento de espécies que necessitam de abertura de clareiras para seu estabelecimento na comunidade, e mantendo aquelas resistentes ao fogo. Embora queimadas frequentes possam reduzir a diversidade funcional de espécies arbóreas (CIANCIARUSO et al., 2010; SILVA et al., 2011), em menor frequência também podem aumentar esta diversidade funcional (CIANCIARUSO et al., 2010), tal como observado no presente estudo. Neste caso, a resistência ao fogo observada em algumas espécies vegetais do Cerrado permite que as comunidades mantenham suas populações (MOREIRA, 2000; HOFFMANN, 2000). Por outro lado, nos casos em que a diversidade funcional é reduzida devido à passagem do fogo, as espécies com pouca ou nenhuma adaptação às queimadas acabam sendo eliminadas da comunidade. Assim, o fogo pode atuar como um filtro ambiental, estruturando as características funcionais (VERDÚ; PAUSAS, 2007; PAUSAS; VERDÚ, 2008) e tendendo a reunir espécies co-ocorrentes em nichos similares, com características funcionais semelhantes (FUKAMI et al., 2005) que geram a redundância funcional (WEBB et al., 2002; CIANCIARUSO et al., 2009). 
O fogo também pode ter favorecido o aumento da riqueza e do número de indivíduos no cerrado típico. Neste caso, sugere-se que a mortalidade de algumas espécies menos resistentes ao fogo (e.g. Tachigali vulgaris e Guapira graciliflora) possa ter favorecido a entrada (alcançado o diâmetro mínimo de inclusão) de outras espécies mais adaptadas, enquanto que, a persistência de outras espécies (e.g. Kielmeyera rubriflora) na comunidade deve-se às adaptações morfológicas específicas para resistência ao fogo (HOFFMANN, 2000; MOREIRA, 2000). Além da capacidade de manter-se na comunidade após incêndios, muitos estudos têm demonstrado que formações vegetais do Cerrado apresentam alta capacidade de resiliência para se reestabelecerem na comunidade (RATTER et al., 2003; MEWS et al., 2011). Assim, a grande capacidade de rebrota de muitas espécies garante uma elevada taxa de recrutamento (HOFFMANN; MOREIRA, 2002; AQUINO et al., 2007) e, portanto, a resiliência.

Os padrões registrados no cerrado típico também sugerem que as mudanças que ocorreram na riqueza e no número de indivíduos da comunidade podem ser caracterizadas pelo balanço positivo entre a entrada e saída de algumas espécies e pelo aumento na densidade de indivíduos, indicando que as mudanças temporais também ocorrem em espécies pouco abundantes (LIBANO; FELFILI, 2006; MEWS et al., 2011). Essas mudanças positivas podem estar relacionadas à ausência de fogo por um período superior a três anos, favorecendo o estabelecimento de muitos indivíduos, compensando a mortalidade e promovendo aumento da densidade (MOREIRA, 2000; HOFFMANN; MOREIRA, 2002; MEWS et al., 2011). Neste caso, a manutenção ou aumento temporal da riqueza é um padrão esperado, especialmente nas fisionomias savânicas do Cerrado (AQUINO et al., 2007; MEWS et al., 2011).

$\mathrm{O}$ aumento da diversidade funcional no cerradão e no cerrado típico reforça o caráter de resiliência destas comunidades, que também pode ter sido promovido pelo aumento na precipitação registrado nos anos de 2003 e 2004, subsequentes ao fogo de 2001. Além disso, as áreas estudadas encontram-se em uma região com forte influência amazônica, apresentam elevados valores de riqueza e diversidade (MARIMON-JUNIOR;
HARIDASAN, 2005; MARIMON et al., 2006) e também são comunidades marcadamente mais dinâmicas quando comparados com outras áreas localizadas em porções mais centrais do Cerrado e da Amazônia (MARIMON et al., 2014). Neste caso, o aumento ou manutenção da diversidade funcional em fisionomias florestais e savânicas da transição Cerrado-Amazônia, em um intervalo de aproximadamente 10 anos, reforça o caráter resiliente destas fisionomias às perturbações, permitindo às comunidades manter e até aumentar sua diversidade funcional mesmo sob ação de eventos de seca e queimadas.

Apesar de a diversidade funcional não ter aumentado, ela permaneceu constante na mata galeria ao longo do período. Neste caso, as condições ambientais mais estáveis na mata de galeria podem ser fatores importantes na manutenção da diversidade funcional ao longo do tempo, especialmente em relação à umidade do solo, pois as espécies têm acesso à água durante todo o ano (MARIMON et al., 2002). Outra explicação para a manutenção da diversidade funcional seria que as espécies incluídas ao longo dos anos são funcionalmente redundantes. Ou seja, apresentaram características funcionais similares às que já se encontravam na comunidade, não contribuindo para uma mudança significativa na diversidade funcional (PETCHEY; GASTON, 2002; 2006) e garantindo a manutenção das funções ecossistêmicas (BLÜTHGEN, 2012).

Considerando que no cerradão e na mata de galeria a diversidade funcional não diferiu do acaso, os processos que independem das características das espécies (e.g. limitação de dispersão e processos neutros) podem ser mais importantes na estruturação das assembleias (PAUSAS; VERDÚ, 2010). Por outro lado, no cerrado típico, onde a diversidade funcional diferiu do acaso em 2011, pode-se inferir que esteja ocorrendo algum processo na estruturação dessa assembleia, com a ocorrência de filtros ambientais que selecionam espécies com características funcionais mais similares e aptas para sobreviver no ambiente (WEIHER; KEDDY, 1995). No caso do cerrado típico, sugere-se que este filtro seja o fogo, visto que queimadas frequentes podem reduzir a diversidade funcional (CIANCIARUSO et al., 2012). 
Em síntese, o fogo pode ter sido o principal fator na alteração dos valores das características funcionais das espécies lenhosas das comunidades de cerrado típico e cerradão, indicando complementaridade funcional das espécies nestas comunidades. O padrão encontrado no cerrado típico sugere que o fogo possa estar selecionando espécies com características funcionais similares. Além disso, mesmo após períodos de seca e eventos de fogo, que levaram à entrada e saída de indivíduos e espécies nas comunidades, a diversidade funcional não diminuiu. Pelo contrário, esta se manteve e até mesmo aumentou ao longo do tempo, indicando a resiliência das comunidades avaliadas. Frente ao cenário atual de intervenções antrópicas e mudanças ambientais que as formações vegetais de Cerrado vêm sofrendo, a continuidade dos estudos das características funcionais das espécies permitirá uma melhor avaliação das estratégias, adaptações, variações e processos ecossistêmicos que mantêm as comunidades em funcionamento.

\section{Agradecimentos}

À CAPES pela bolsa concedida à primeira autora; ao $\mathrm{CNPq} / \mathrm{PELD}$ e CNPq/PPBio pelo apoio financeiro (Proj. no 558069/2009-6 e 457602/2012-0) e ao CNPq, pela bolsa de produtividade em pesquisa de BSM e BHMJr. Aos amigos, professores e colegas do Laboratório de Ecologia Vegetal (LABEV), pelo auxílio na coleta de dados no campo e ao Prof. Dr. Marcus Cianciaruso (UFG), pelo auxílio nas análises estatísticas.

\section{Referências}

ABAD, J. C. S.; MARIMON, B. S. Caracterização e diagnóstico ambiental do Parque Municipal do Bacaba (Nova Xavantina, MT). In: SANTOS, J. E.; GALBIATI, C. (Org.). Gestão e educação ambiental: água, biodiversidade e cultura. Rima: São Carlos, 2008. p. 23-56.

AQUINO, F. G.; WALTER, B. M. T.; RIBEIRO, J. F. Woody community dynamics in two fragments of "cerrado" stricto sensu over a seven year period (1995-2002). Revista Brasileira de Botânica, São Paulo, v. 30, p. 113-121, 2007.

BARROSO, G. M.; MORRIM, M. P.; PEIXOTO, A. L.; ICHASO, C. L. F. Frutos e sementes. Morfologia aplicada à sistemática de Dicotiledôneas. Viçosa: EdUFV, 1999. 443 p.

BATALHA, M. A.; SILVA, I. A. CIANCIARUSO, M. V.; CARVALHO, G. H. Trait diversity on the phylogeny of cerrado woody species. Oikos, Malden, v. 120, n. 11, p. 1741-1751, 2011.
BLÜTHGEN, N. Interações plantas-animais e a importância funcional da biodiversidade. In: DEL-CLARO, K.; TOREZANSILINGARDI, H. M. (Ed.). Ecologia das interações plantas animais: uma abordagem ecológico-evolutiva. Rio de Janeiro: Technical Books, 2012. p. 261-304.

BROWER, J. E.; ZAR, J. H. Field and laboratory methods for general ecology. Iowa: W.C. Brown Co. Pub., 1977. 226 p.

CARVALHO, P. E. R. Espécies arbóreas brasileiras. Brasília: EMBRAPA, 2003. 1038 p.

CIANCIARUSO, M. V.; BATALHA, M. A.; GASTON, K. J.; PETCHEY, O. L. Including intraspecific variability in functional diversity. Ecology, Ithaca, v. 90, p. 81-89, 2009.

CIANCIARUSO, M. V.; SILVA, I. A.; BATALHA, M. A. Aboveground biomass of functional groups in the ground layer of savannas under different fire frequencies. Australian Journal of Botany, Collingwood, v. 58, p. 169-174, 2010.

CIANCIARUSO, M. V.; SILVA, I.A.; BATALHA, M.A.; GASTON, K. J.; PETCHEY, O. L. The influence of fire on phylogenetic and functional structure of woody savannas: Moving from species to individuals. Perspectives in plant ecology, evolution and systematics, Jena, v. 14, p. 205-216, 2012.

CIANCIARUSO, M. V.; SILVA, I. A.; MANICA, L. T. SOUZA, J. P. Leaf habit does not predict leaf functional traits in cerrado woody species. Basic and Applied Ecology, Jena, v. 14, p. 404-412, 2013. CORNELISSEN, J. H. C.; LAVOREL, S.; GARNIEL, E.; DÍAZ, S.; BUCHMANN, N.; GUREVICH, D. E.; REICH, P. B.; TER STEEGE, H.; MORGAN, H. D.; HEIJDEN, M. G. A.; PAUSAS, J. G.; POORTER, H. A handbook of protocols for standardised and easy measurement of plant functional traits worldwide. Australian Journal of Botany, Collingwood, v. 51, p. 335-380, 2003.

COUTINHO, L. M. Fire in the ecology of the Brazilian Cerrado. In: GOLDAMMER J. G. (Ed.). Fire in the tropical biota. Berlin: Springer, p. 81-103, 1990.

DANTAS, V. L.; PAUSAS, J. G. BATALHA, M. A.; LOIOLA, P. P.; CIANCIARUSO, M. V. The role of fire in structuring trait variability in Neotropical savannas. Oecologia, New York, v. 171, p. 487-494, 2013.

ELIAS, F.; MARIMON, B. S.; GOMES, L.; FORSTHOFER, M.; ABREU, M. F.; REIS, S. A.; LENZA, E.; FRANCZAK, D. D.; MARIMON-JUNIOR, B. H. Resiliência de um cerradão submetido a perturbações intermediárias na transição Cerrado-Amazônia. Biotemas, Florianópolis, v. 26, n. 3, p. 49-62, 2013.

FRANCZAK, D. D.; MARIMON, B. S.; MARIMON-JUNIOR, B. H.; MEWS, H. A.; MARACAHIPES, L.; OLIVEIRA E. A. Changes in the structure of a savanna forest over a six-year period in the Amazon-Cerrado transition, Mato Grosso state, Brazil. Rodriguésia, Rio de Janeiro, v. 62, n. 2, p. 425-436, 2011.

FUKAMI, T.; BEZEMER, T. M.; MORTIMER, S. R.; VAN DER PUTTEN, W. H. Species divergence and trait convergence in experimental plant community assembly. Ecology Letters, London, v. 8, n. 12, p. 1283-1290, 2005.

HOFFMANN, W. A. Post-establishment seedling success of savanna and forest species in the Brazilian Cerrado. Biotropica, Malden, v. 32, p. 62-69, 2000.

HOFFMANN, W. A. Ecologia comparativa de espécies lenhosas de cerrado e mata. In. SCARIOT, A.; SOUSA-SILVA, J. C.; FELFILI, 
J. M. (Ed.). Cerrado: ecologia, biodiversidade e conservação. Brasília: MMA/EdUnB, 2005. p. 157-165.

HOFFMANN, W. A.; MOREIRA, A. G. The role of fire in population dynamics of woody plants. In: OLIVEIRA, P. S.; MARQUIS, R. J. (Eds.). The Cerrados of Brazil: ecology and natural history of a Neotropical savanna. New York: Columbia University Press, 2002. p. $159-177$.

KEMBEL, S. W. Disentangling niche and neutral influences on community assembly: assessing the performance of community phylogenetic structure tests. Ecology Letters, London, v. 12, p. 949-960, 2009.

KENT, M.; COKER, P. Vegetation description and analysis: a practical approach. London: Belhaven Press, 1992. 363 p.

KLINK, C. A.; MACHADO, R. B. A conservação do Cerrado brasileiro. Megadiversidade, Belo Horizonte, v. 1, p. 147-155, 2005.

LEWIS, S. L.; BRANDO, P. M.; PHILLIPS, O. L.; HEIJDEN, G. M. F.; NEPSTAD, D. The 2010 Amazon Drought. Science, Washington, v. 331, p. 554-555, 2011.

LIBANO, A. M.; FELFILI, J. M. Mudanças temporais na composição florística e na diversidade de um cerrado sensu stricto do Brasil Central em um período de 18 anos (1985 - 2003). Acta Botanica Brasilica, Feira de Santana, v. 20, n. 4, p. 927-936, 2006.

LORENZI, H. Árvores brasileiras: manual de identificação e cultivo de plantas arbóreas nativas do Brasil. Vol.1. 4. ed. Nova Odessa, SP: Instituto Plantarum, 1992. 384 p.

LORENZI, H. Árvores brasileiras: manual de identificação e cultivo de plantas arbóreas nativas do Brasil. Vol. 2. 2. ed. Nova Odessa: Instituto Plantarum, 1998. 384 p.

MARENGO, J. A.; NOBRE, C. A.; TOMASELLA, J. The Drought of Amazonia in 2005. Journal of Climate, Washington, v. 21, p. 495-516, 2008.

MARIMON, B. S.; FELFILI, J. M.; LIMA, E. S. Floristic and phytosociology of the gallery forest of the Bacaba stream, Nova Xavantina, Mato Grosso, Brazil. Edinburgh Journal of Botany, Edinburgh, v. 59, n. 2, p. 303-318, 2002.

MARIMON, B. S.; FELFILI, J. M.; LIMA, E. S.; DUARTE, W. M. G.; MARIMON-JUNIOR, B. H. Environmental determinants for natural regeneration of gallery forest at the Cerrado/Amazonia boundaries in Brazil. Acta Amazonica, Manaus, v. 40, p. 107-118, 2010.

MARIMON, B. S.; FELFILI, J. M.; LIMA, E. S.; RODRIGUES, A. J. Distribuição de circunferências e alturas em três porções da mata de galeria do Córrego Bacaba, Nova Xavantina - MT. Revista Árvore, Viçosa, v. 25, n. 3, p. 335-343, 2001.

MARIMON, B. S.; LIMA, E. S.; DUARTE, T. G.; CHIEREGATTO, L. C.; RATTER, J. A. Observations on the vegetation of northeastern Mato Grosso, Brazil. IV. An analysis of the Cerrado-Amazonian forest ecotone. Edinburgh Journal of Botany, Edinburgh, v. 63, p. 323-341, 2006.

MARIMON, B. S.; MARIMON-JUNIOR, B. H.; FELDPAUSCH, T. R.; OLIVEIRA-SANTOS, C.; MEWS, H. A.; LOPEZGONZALEZ, G.; LLOYD, J.; FRANCZAK, D. D.; OLIVEIRA, E. A.; MARACAHIPES, L.; MIGUEL, A.; LENZA, E.; PHILLIPS, O. L. Disequilibrium and hyperdynamic tree turnover at the forestsavanna transition zone in southern Amazonia. Plant Ecology and Diversity, Edinburgh, v. 7, p. 281-292, 2014.
MARIMON-JUNIOR, B. H.; HARIDASAN, M. Comparação da vegetação arbórea e características edáficas de um cerradão e um cerrado sensu stricto em áreas adjacentes sobre solo distrófico no leste de Mato Grosso, Brasil. Acta Botanica Brasilica, Feira de Santana, v. 19, n. 4, p. 913-926, 2005.

MAYFIELD, M. M.; ACKERLY, D.; DAILY G. C. The diversity and conservation of plant reproductive and dispersal functional traits in human-dominated tropical landscapes. Journal of Ecology, London, v. 94, p. 522-536, 2006.

MCEWEN, J. R.; VAMOSI, J. C. Floral colour versus phylogeny in structuring subalpine flowering communities. Proceedings of the Royal Society of London, Series Biological Sciences, London, v. 277, p. 2957-2965, 2010.

MEWS, H. A.; MARIMON, B. S.; MARACAHIPES, L.; FRANCZAK, D. D.; MARIMON-JUNIOR, B. H. Dinâmica da comunidade lenhosa de um cerrado típico na região nordeste do estado de Mato Grosso, Brasil. Biota Neotropica, Campinas, v. 11, n. 1, p. 73-82, 2011.

MIGUEL, A.; MARIMON, B. S.; MARACAHIPES, L.; OLIVEIRA, E. A.; MARIMON-JUNIOR, B. H. Mudanças na estrutura da vegetação lenhosa em três porções da mata de galeria do Córrego Bacaba (1999-2006), Nova Xavantina-MT. Revista Árvore, Viçosa, v. 35, n. 3, p. 725-735, 2011 b.

MIGUEL, A.; MARIMON, B. S.; OLIVEIRA, E. A.; MARACAHIPES, L.; MARIMON-JUNIOR, B. H. Dinâmica da comunidade lenhosa de uma floresta de galeria na transição Cerrado-Floresta Amazônica no Leste de Mato Grosso, em um período de sete anos (1999 a 2006). Biota Neotropica, Campinas, v. 11, n. 1, p. 53-61, 2011a.

MOREIRA, A. G. Effects of fire protection on savanna structure in Central Brazil. Journal of Biogeography, Malden, v. 27, p. 10211029, 2000.

MOUCHET, M.; VILLÉGER, S.; MASON, N. W. H.; MOUILLOT, D. Functional diversity measures: an overview of their redundancy and their ability to discriminate community assembly rules. Functional Ecology, London, v. 24, p. 867-876, 2010.

OLIVEIRA, P. E. A. M. Fenologia e biologia reprodutiva das espécies de Cerrado. In: SANO, S. M.; ALMEIDA, S. P.; RIBEIRO, J. F. (Ed.). Cerrado: ecologia e flora. Brasília: EMBRAPA, 2008. p. 273-287.

PAUSAS, J. G.; VERDÚ, M. Fire reduces morphospace occupation in plant communities. Ecology, London, v. 89, p. 2181-2186, 2008. PAUSAS, J. G.; VERDÚ, M. The jungle of methods for evaluating phenotypic and phylogenetic structure of communities. BioScience, Berkeley, v. 60, n. 8, p. 614-625, 2010.

PAVOINE, S.; BONSALL, M. B. Measuring biodiversity to explain community assembly: a unified approach. Biological Reviews, Cambridge, v. 86, n. 4, p. 792-812, 2011.

PAVOINE, S.; VALLET, J.; DUFOUR, A. B.; GACHET, S.; DANIEL, H. On the challenge of treating various types of variables: application for improving the measurement of functional diversity. Oikos, Malden, v. 118, p. 391-402, 2009.

PETCHEY, O. L.; GASTON, K. J. Functional diversity (FD), species richness, and community composition. Ecology Letters, London, v. 5, n. 3, p. 402-411, 2002. 
PETCHEY, O. L.; GASTON, K. J. Functional diversity: back to basics and looking forward. Ecology Letters, London, v. 9, n. 6, p. 741-758, 2006.

R DEVELOPMENT CORE TEAM. A language and environment for statistical computing. URL http://www.R-project.org [2.10.1]. Vienna: R Foundation for Statistical Computing, 2012.

RATTER, J. A.; BRIDGEWATER, S.; RIBEIRO, J. F. Analysis of the floristic composition of the Brazilian cerrado vegetation III: comparison of the woody vegetation of 376 areas. Edinburgh Journal of Botany, Edinburgh, v. 60, p. 57-109, 2003.

SILVA, I. A.; LOIOLA, P. P.; ROSATTI, N. B.; SILVA, I. A.; CIANCIARUSO, M. V.; BATALHA, M. A. Os efeitos dos regimes de fogo sobre a vegetação de Cerrado no Parque Nacional das Emas, GO: considerações para a conservação da diversidade. Biodiversidade Brasileira, Brasília, v. 2, p. 26-39, 2011.
STATSOFT, INC. Statistica 7.0. L http://www.statsoft.com, 2007. VERDÚ, M.; PAUSAS, J. G. Fire drives phylogenetic clustering in Mediterranean Basin woody plant communities. Journal of Ecology, London, v. 95, p. 1316-1323, 2007.

WEBB, C. O. Exploring the phylogenetic structure of ecological communities: an example for rain forest trees. The American Naturalist, Chicago, v. 156, n. 2, p. 145-155, 2000.

WEBB, C. O.; ACKERLY, D. D.; MCPEEK, M. A.; DONOGHUE, M. J. Phylogenies and community ecology. Annual Review of Ecology and Systematics, Palo Alto, v. 33, p. 475-505, 2002.

WEIHER, E.; KEDDY, P. A. Assembly rules, null models, and trait dispersion: new questions from old patterns. Oikos, Malden, v. 74, n. 1, p. 159-164, 1995. 